\title{
Stability for a Holling Type IV Food Chain System With Im- pulsive Perturbations
}

Hunki BAEK AND Younghae Do

Department of Mathematics, Kyungpook National University, Daegu 702-701, South

Korea

e-mail : hkbaek@knu.ac.kr and yhdo@knu.ac.kr

ABSTRACT. We investigate a three species food chain system with a Holling type IV functional response and impulsive perturbations. We find conditions for local and global stabilities of prey(or predator) free periodic solutions by applying the Floquet theory and the comparison theorems.

\section{Introduction}

It is currently very much in vogue to study population models with impulsive perturbations containing biological and chemical controls. Especially, simple multispecies systems consisting of a three species food chain with impulsive perturbations have been discussed by a number of researchers [13], [17], [18], [19], [20] and there are also many literatures on impulsive prey-predator population models [10], [11], $[12]$.

A well-known model of such systems is a food chain system with Holling type IV functional response [7], [14], [20], which can be described the following equation:

$$
\left\{\begin{array}{l}
x^{\prime}(t)=x(t)(a-b x(t))-\frac{c_{1} x(t) y(t)}{1+e_{1} x^{2}(t)} \\
y^{\prime}(t)=-d_{1} y(t)+\frac{c_{2} x(t) y(t)}{1+e_{1} x^{2}(t)}-\frac{c_{3} y(t) z(t)}{1+e_{2} y^{2}(t)} \\
z^{\prime}(t)=-d_{2} z(t)+\frac{c_{4} y(t) z(t)}{1+e_{2} y^{2}(t)}
\end{array}\right.
$$

where $x(t), y(t), z(t)$ are the densities of the lowest-level prey, mid-level predator and top predator at time $t$, respectively, $a, b, c_{1}, c_{2}, c_{3}, c_{4}, d_{1}, d_{2}, e_{1}$ and $e_{2}$ are positive constants. In this paper, we consider an impulsive differential equation - a three species food chain system with Holling type IV functional response, by introducing a proportion periodic impulsive poisoning (chemical control) for all species and a constant periodic releasing, or immigrating, (biological control) for the top predator

Received June 17, 2008.

2000 Mathematics Subject Classification: 34A37,34D23,34H05,92D25.

Key words and phrases: three-species food chain systems, Holling type IV functional response, impulsive differential equations, Floquet thoery 
at different fixed time. Thus, we establish a food chain system with impulsive perturbations as follows:

$(1.2)$

$$
\left\{\begin{array}{l}
x^{\prime}(t)=x(t)(a-b x(t))-\frac{c_{1} x(t) y(t)}{1+e_{1} x^{2}(t)} \\
\left.y^{\prime}(t)=-d_{1} y(t)+\frac{c_{2} x(t) y(t)}{1+e_{1} x^{2}(t)}-\frac{c_{3} y(t) z(t)}{1+e_{2} y^{2}(t)},\right\} t \neq n T, t \neq(n+\tau-1) T \\
z^{\prime}(t)=-d_{2} z(t)+\frac{c_{4} y(t) z(t)}{1+e_{2} y^{2}(t)} \\
x\left(t^{+}\right)=\left(1-p_{1}\right) x(t), \\
y\left(t^{+}\right)=\left(1-p_{2}\right) y(t), \\
z\left(t^{+}\right)=\left(1-p_{3}\right) z(t), \\
x\left(t^{+}\right)=x(t) \\
y\left(t^{+}\right)=y(t), \\
z\left(t^{+}\right)=z(t)+q, \\
\left(x\left(0^{+}\right), y\left(0^{+}\right), z\left(0^{+}\right)\right)=\left(x_{0}, y_{0}, z_{0}\right)
\end{array}\right.
$$

where $0 \leq \tau, p_{1}, p_{2}, p_{3}<1$ and $T$ is the period of the impulsive immigration or stock the top predator and $q$ is the size of immigration or stock of the top predator. Theories and applications for impulsive differential equations were greatly developed by the efforts of Bainov and Lakshmikantham et al. [2], [9] and, moreover, the theory of impulsive differential equations is being recognized to be not only richer than the corresponding theory of differential equations without impulses, but also represents a more natural framework for mathematical modeling of real world phenomenons.

The authors in [13] and [19] have studied the stabilities for a food chain system with Holling type II and impulsive perturbations. Especially, the authors in [20] have studies the local stability of a lower-level prey and mid-level predator free periodic solution and a mid-level predator free periodic solution of the system (1.2) with $p_{1}=p_{2}=p_{3}=0$.

The rest of the paper is organized as follows: In the next section, we introduce some notations and lemmas which are used in this paper. In section 3, we find conditions for local and global stabilities of a lower-level prey and a mid-level predator free periodic solution and mid-level predator free periodic solution by applying the Floquet theory and comparison theorems.

\section{Preliminaries}

First, we shall introduce a few notations and definitions together with a few auxiliary results relating to comparison theorems, which will be useful for our main results.

Let $\mathbb{R}_{+}=[0, \infty)$ and $\mathbb{R}_{+}^{3}=\left\{\mathbf{x}=(x(t), y(t), z(t)) \in \mathbb{R}^{3}: x(t), y(t), z(t) \geq 0\right\}$. Denote $\mathbb{N}$ the set of all of nonnegative integers, $\mathbb{R}_{+}^{*}=(0, \infty)$ and $f=\left(f_{1}, f_{2}, f_{3}\right)^{T}$ the right hand of the first three equations in (1.2). Let $V: \mathbb{R}_{+} \times \mathbb{R}_{+}^{3} \rightarrow \mathbb{R}_{+}$. Then 
$V$ is said to be in a class $V_{0}$ if

(1) $V$ is continuous on $(n T,(n+1) T] \times \mathbb{R}_{+}^{3}$, and $\lim _{\substack{(t, \mathbf{y}) \rightarrow(n T, \mathbf{x}) \\ t>n T}} V(t, \mathbf{y})=V\left(n T^{+}, \mathbf{x}\right)$ exists.

(2) $V$ is a local Lipschitzian in $\mathbf{x}$.

Definition 2.1. For $V \in V_{0}$, we define the upper right Dini derivative of $V$ with respect to the impulsive differential system $(1.2)$ at $(t, \mathbf{x}) \in(n T,(n+1) T] \times \mathbb{R}_{+}^{3}$ by

$$
D^{+} V(t, \mathbf{x})=\limsup _{h \rightarrow 0+} \frac{1}{h}[V(t+h, \mathbf{x}+h f(t, \mathbf{x}))-V(t, \mathbf{x})] .
$$

Remark 2.2. The smoothness properties of $f$ guarantee the global existence and uniqueness of solutions of the system (1.2). (See [9] for the details).

We will use a comparison result of impulsive differential inequalities. We suppose that $g: \mathbb{R}_{+} \times \mathbb{R}_{+} \rightarrow \mathbb{R}$ satisfies the following hypothesis:

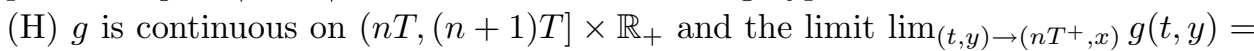
$g\left(n T^{+}, x\right)$ exists and is finite for $x \in \mathbb{R}_{+}$and $n \in \mathbb{N}$.

Lemma 2.3 ([9]). Suppose $V \in V_{0}$ and

$$
\left\{\begin{array}{l}
D^{+} V(t, \mathbf{x}) \leq g(t, V(t, \mathbf{x})), t \neq(n+\tau-1) T, t \not h T \\
V\left(t, \mathbf{x}\left(t^{+}\right)\right) \leq \psi_{n}^{1}(V(t, \mathbf{x})), t=(n+\tau-1) T \\
V\left(t, \mathbf{x}\left(t^{+}\right)\right) \leq \psi_{n}^{2}(V(t, \mathbf{x})), t=n T
\end{array}\right.
$$

where $g: \mathbb{R}_{+} \times \mathbb{R}_{+} \rightarrow \mathbb{R}$ satisfies $(H)$ and $\psi_{n}^{1}, \psi_{+}^{2}: \mathbb{R}_{+} \rightarrow \mathbb{R}_{+}$are non-decreasing for all $n \in \mathbb{N}$. Let $r(t)$ be the maximal solution for the impulsive Cauchy problem

$$
\left\{\begin{array}{l}
u^{\prime}(t)=g(t, u(t)), t \neq(n+\tau-1) T, t h T \\
u\left(t^{+}\right)=\psi_{n}^{1}(u(t)), t=(n+\tau-1) T \\
u\left(t^{+}\right)=\psi_{n}^{2}(u(t)), t=n T \\
u\left(0^{+}\right)=u_{0}
\end{array}\right.
$$

defined on $[0, \infty)$. Then $V\left(0^{+}, \mathbf{x}_{0}\right) \leq u_{0}$ implies that $V(t, \mathbf{x}(t)) \leq r(t), t \geq 0$, where $\mathbf{x}(t)$ is any solution of $(2.1)$.

We now indicate a special case of Lemma 2.3, which provides estimations for the solution of a system of differential inequalities. For this, we let $P C\left(\mathbb{R}_{+}, \mathbb{R}\right)\left(P C^{1}\left(\mathbb{R}_{+}, \mathbb{R}\right)\right)$ denote the class of real piecewise continuous(real piecewise continuously differentiable) functions defined on $\mathbb{R}_{+}$. 
Lemma $2.4([9])$. Let the function $u(t) \in P C^{1}\left(\mathbb{R}^{+}, \mathbb{R}\right)$ satisfy the inequalities

$$
\left\{\begin{aligned}
\frac{d u}{d t} & \leq f(t) u(t)+h(t), t \neq \tau_{k}, t>0 \\
u\left(\tau_{k}^{+}\right) & \leq \alpha_{k} u\left(\tau_{k}\right)+\beta_{k}, k \geq 0 \\
u\left(0^{+}\right) & \leq u_{0}
\end{aligned}\right.
$$

where $f, h \in P C\left(\mathbb{R}_{+}, \mathbb{R}\right)$ and $\alpha_{k} \geq 0, \beta_{k}$ and $u_{0}$ are constants and $\left(\tau_{k}\right)_{k \geq 0}$ is a strictly increasing sequence of positive real numbers. Then, for $t>0$,

$$
\begin{aligned}
u(t) \leq & u_{0}\left(\prod_{0<\tau_{k}<t} \alpha_{k}\right) \exp \left(\int_{0}^{t} f(s) d s\right)+\int_{0}^{t}\left(\prod_{0 \leq \tau_{k}<t} d_{k}\right) \exp \left(\int_{s}^{t} f(\gamma) d \gamma\right) h(s) d s \\
& +\sum_{0<\tau_{k}<t}\left(\prod_{\tau_{k}<\tau_{j}<t} d_{j}\right) \exp \left(\int_{\tau_{k}}^{t} f(\gamma) d \gamma\right) \beta_{k} .
\end{aligned}
$$

Similar result can be obtained when all conditions of the inequalities in the Lemma 2.3 and 2.4 are reversed. Using Lemma 2.4, it is possible to prove that the solutions of the Cauchy problem (2.2) with strictly positive initial value remain strictly positive.

Lemma 2.5. The positive orthant $\left(\mathbb{R}_{+}^{*}\right)^{3}$ is an invariant region for the system (1.2).

Proof. Let $(x(t), y(t), z(t)):\left[0, t_{0}\right) \rightarrow \mathbb{R}^{2}$ be a saturated solution of the system (1.2) with a strictly positive initial value $\left(x_{0}, y_{0}, z_{0}\right)$. It is easy to see that, for $t n \neq(n+\tau-1) T, t \neq n T$ and $0 \leq t<t_{0}$,

$$
\left\{\begin{array}{l}
x^{\prime}(t) \geq x(t)(a-b x(t))-c_{1} x(t) y(t) \\
y^{\prime}(t) \geq-d_{1} y(t)-c_{3} y(t) z(t) \\
z^{\prime}(t) \geq-d_{2} z(t)
\end{array}\right.
$$

as long as the solution remains positive. By Lemma 2.4, we can obtain that, for $0 \leq t<t_{0}$

$$
\left\{\begin{array}{l}
x(t) \geq x_{0}\left(1-p_{1}\right)^{\left[\frac{t}{T}\right]} \exp \left(\int_{0}^{t} f_{1}(s) d s\right) \\
y(t) \geq y_{0}\left(1-p_{2}\right)^{\left[\frac{t}{T}\right]} \exp \left(\int_{0}^{t} f_{2}(s) d s\right) \\
z(t) \geq z_{0}\left(1-p_{3}\right)^{\left[\frac{t}{T}\right]} \exp \left(\int_{0}^{t} f_{3}(s) d s\right)
\end{array}\right.
$$

where $f_{1}(s)=a-b x(s)-c_{1} y(s), f_{2}(s)=-d_{1}-c_{3} z(s)$ and $f_{3}(s)=-d_{2}$. Thus, $x(t), y(t)$ and $z(t)$ remain strictly positive on $\left[0, t_{0}\right)$. 
Now, we give the basic properties of two impulsive differential equations. First, we consider the following impulsive differential equation.

$$
\left\{\begin{array}{l}
x^{\prime}(t)=x(t)(a-b x(t)), t \neq n T, t \neq(n+\tau-1) T, \\
x\left(t^{+}\right)=\left(1-p_{1}\right) x(t), t=(n+\tau-1) T, \\
x\left(t^{+}\right)=x(t), t=n T, \\
x\left(0^{+}\right)=x_{0} .
\end{array}\right.
$$

The system (2.6) is a periodically forced system. It is easily obtain that

(2.7) $x^{*}(t)=\frac{a \eta \exp (a(t-(n+\tau-1) T))}{b(1-\eta+\eta \exp (a(t-(n+\tau-1) T)))},(n+\tau-1) T<t \leq(n+\tau) T$,

is a positive periodic solution of $(2.6)$, where $\eta=\frac{\left(1-p_{1}\right) \exp (a T)-1}{\exp (a T)-1}$. Now, we mention the following Lemma in [13].

Lemma 2.6 ([13]). The following statements hold.

(1) If $a T+\ln \left(1-p_{1}\right)>0$, then $\lim _{t \rightarrow \infty}\left|x(t)-x^{*}(t)\right|=0$ for all solutions $x(t)$ of (2.6) with $x_{0}>0$.

(2) If aT $+\ln \left(1-p_{1}\right) \leq 0$, then $x(t) \rightarrow 0$ as $t \rightarrow \infty$ for all solutions $x(t)$ of (2.6).

Next, we consider the impulsive differential equation as follows:

$$
\left\{\begin{array}{l}
z^{\prime}(t)=-d_{2} z(t), t \neq n T, t \neq(n+\tau-1) T, \\
z\left(t^{+}\right)=\left(1-p_{3}\right) z(t), t=(n+\tau-1) T, \\
z\left(t^{+}\right)=z(t)+q, t=n T, \\
z\left(0^{+}\right)=z_{0} .
\end{array}\right.
$$

The system (2.8) is a periodically forced linear system. It is easy to obtain that

$$
z^{*}(t)=\left\{\begin{array}{l}
\frac{q \exp \left(-d_{2}(t-(n-1) T)\right)}{1-\left(1-p_{3}\right) \exp \left(-d_{2} T\right)},(n-1) T<t \leq(n+\tau-1) T, \\
\frac{q\left(1-p_{3}\right) \exp \left(-d_{2}(t-(n-1) T)\right)}{1-\left(1-p_{3}\right) \exp \left(-d_{2} T\right)},(n+\tau-1) T<t \leq n T,
\end{array}\right.
$$

$z^{*}\left(0^{+}\right)=z^{*}\left(n T^{+}\right)=\frac{q}{1-\left(1-p_{3}\right) \exp \left(-d_{2} T\right)}, z^{*}\left((n+\tau-1) T^{+}\right)=\frac{q\left(1-p_{3}\right) \exp \left(-d_{2} \tau T\right)}{1-\left(1-p_{3}\right) \exp \left(-d_{2} T\right)}$ is a positive periodic solution of (2.8).

Moreover, we can obtain that

$$
z(t)=\left\{\begin{array}{l}
\left(1-p_{3}\right)^{n-1}\left(z\left(0^{+}\right)-\frac{q\left(1-p_{3}\right) e^{-T}}{1-\left(1-p_{3}\right) \exp \left(-d_{2} T\right)}\right) \exp \left(-d_{2} t\right)+z^{*}(t), \\
(n-1) T<t \leq(n+\tau-1) T \\
\left(1-p_{3}\right)^{n}\left(z\left(0^{+}\right)-\frac{q\left(1-p_{3}\right) e^{-T}}{1-\left(1-p_{3}\right) \exp \left(-d_{2} T\right)}\right) \exp \left(-d_{2} t\right)+z^{*}(t), \\
(n+\tau-1) T<t \leq n T,
\end{array}\right.
$$


is a solution of (2.8). From (2.9) and (2.10), we get easily the following result.

Lemma 2.7. All solutions $z(t)$ of (1.2) tend to $z^{*}(t)$. i.e., $\left|z(t)-z^{*}(t)\right| \rightarrow 0$ as $t \rightarrow \infty$.

To study the stability of the lowest-level prey and top predator free periodic solution $\left(0,0, z^{*}(t)\right)$ and of the mid-level predator free periodic solution $\left(x^{*}(t), 0, z^{*}(t)\right)$ we present the Floquet theory for the linear $T$-periodic impulsive equation:

$$
\left\{\begin{array}{l}
x^{\prime}(t)=A(t) x(t), t \neq \tau_{k}, t \in \mathbb{R}, \\
x\left(t^{+}\right)=x(t)+B_{k} x(t), t=\tau_{k}, k \in \mathbb{Z} .
\end{array}\right.
$$

Then we introduce the following conditions:

$\left(H_{1}\right) A(\cdot) \in P C\left(\mathbb{R}, C^{n \times n}\right)$ and $A(t+T)=A(t)(t \in \mathbb{R})$, where $P C\left(\mathbb{R}, C^{n \times n}\right)$ is a set of all piecewise continuous matrix functions which is left continuous at $t=\tau_{k}$, and $C^{n \times n}$ is a set of all $n \times n$ matrices.

$\left(H_{2}\right) B_{k} \in C^{n \times n}, \operatorname{det}\left(E+B_{k}\right) \neq 0, \tau_{k}<\tau_{k+1}(k \in \mathbb{Z})$.

$\left(H_{3}\right)$ There exists a $q \in \mathbb{N}$ such that $B_{k+q}=B_{k}, \tau_{k+q}=\tau_{k}+T(k \in \mathbb{Z})$.

Let $\Phi(t)$ be a fundamental matrix of (2.11), then there exists a unique nonsingular matrix $M \in C^{n \times n}$ such that

$$
\Phi(t+T)=\Phi(t) M(t \in \mathbb{R}) .
$$

By equality (2.12) there corresponds to the fundamental matrix $\Phi(t)$ and the constant matrix $M$ which we call the monodromy matrix of (2.11) (corresponding to the fundamental matrix of $\Phi(t))$.

All monodromy matrices of (2.11) are similar and have the same eigenvalues. The eigenvalues $\mu_{1}, \cdots, \mu_{n}$ of the monodromy matrices are called the Floquet multipliers of (2.11).

Lemma $2.6([2])$. Let conditions $\left(H_{1}\right)-\left(H_{3}\right)$ hold. Then the linear T-periodic impulsive equation (2.11) is

(1) stable if and only if all multipliers $\mu_{j}(j=1, \cdots, n)$ of (2.11) satisfy the inequality $\left|\mu_{j}\right| \leq 1$, and moreover, to those $\mu_{j}$ for which $\left|\mu_{j}\right|=1$, there correspond simple elementary divisors;

(2) asymptotically stable if and only if all multipliers $\mu_{j}(j=1, \cdots, n)$ of $(2.11)$ satisfy the inequality $\left|\mu_{j}\right|<1$;

(3) unstable if $\left|\mu_{j}\right|>1$ for some $j=1, \cdots, n$.

\section{Main theorems}

In this section, we study the stability of the lowest-level prey and mid-level predator free periodic solution $\left(0,0, z^{*}(t)\right)$ and of the mid-level predator free periodic solution $\left(x^{*}(t), 0, z^{*}(t)\right)$. We also show that all solutions of the system (1.2) are uniformly upper bounded.

First, we show that all solutions of (1.2) are uniformly ultimately bounded. 
Theorem 3.1. There is an $M>0$ such that $x(t) \leq M, y(t) \leq M$ and $z(t) \leq M$ for all $t$ large enough, where $(x(t), y(t), z(t))$ is a solution of the system (1.2).

Proof. Let $(x(t), y(t), z(t))$ be a solution of $(1.2)$ and let $u(t)=\frac{c_{2}}{c_{1}} x(t)+y(t)+\frac{c_{3}}{c_{4}} z(t)$ for $t \geq 0$. Then, if $t \neq n T, t \neq(n+\tau-1) T$, then we obtain that

$$
\frac{d u(t)}{d t}=-\frac{c_{2} b}{c_{1}} x^{2}(t)+\frac{c_{2} a}{c_{1}} x(t)-d_{1} y(t)-\frac{c_{3} d_{2}}{c_{4}} z(t) .
$$

From choosing $0<\beta_{0}<\min \left\{d_{1}, d_{2}\right\}$, we obtain

$$
\frac{d u(t)}{d t}+\beta_{0} u(t) \leq-\frac{c_{2} b}{c_{1}} x^{2}(t)+\frac{c_{2}}{c_{1}}\left(a+\beta_{0}\right) x(t), t \neq n T, t \neq(n+\tau-1) T .
$$

As the right-hand side of (3.2) is bounded from above by $M_{0}=\frac{c_{2}\left(a+\beta_{0}\right)^{2}}{4 b c_{1}}$, it follows that

$$
\frac{d u(t)}{d t}+\beta_{0} u(t) \leq M_{0}, t \neq n T, n \neq(n+\tau-1) T .
$$

If $t=n T$, then $u\left(t^{+}\right)=u(t)+\frac{c_{3}}{c_{4}} q$ and if $t=(n+\tau-1) T$, then $u\left(t^{+}\right) \leq(1-p) u(t)$, where $p=\min \left\{p_{1}, p_{2}, p_{3}\right\}$. From Lemma 2.4, we get that

$$
\begin{aligned}
u(t) \leq & u_{0}\left(\prod_{0<k T<t}(1-p)\right) \exp \left(\int_{0}^{t}-\beta_{0} d s\right) \\
& +\int_{0}^{t}\left(\prod_{0 \leq k T<t}(1-p)\right) \exp \left(\int_{s}^{t}-\beta_{0} d \gamma\right) M_{0} d s \\
& +\sum_{0<k T<t}\left(\prod_{k T<j T<t}(1-p)\right) \exp \left(\int_{k T}^{t}-\beta_{0} d \gamma\right) \frac{c_{3}}{c_{4}} q \\
\leq & u\left(0^{+}\right) \exp \left(-\beta_{0} t\right)+\frac{M_{0}}{\beta_{0}}\left(1-\exp \left(-\beta_{0} t\right)\right)+\frac{c_{3} q \exp \left(\beta_{0} T\right)}{c_{4} \exp \left(\beta_{0} T\right)-1} .
\end{aligned}
$$

Since the limit of the right-hand side of (3.3) as $t \rightarrow \infty$ is

$$
\frac{M_{0}}{\beta_{0}}+\frac{c_{3} q \exp \left(\beta_{0} T\right)}{c_{4} \exp \left(\beta_{0} T\right)-1}<\infty,
$$

it easily follows that $u(t)$ is bounded for sufficiently large $t$. Therefore, $x(t), y(t)$ and $z(t)$ are bounded by a constant $M$ for sufficiently large $t$.

Theorem 3.2. The following statements hold.

(1) The periodic solution $\left(0,0, z^{*}(t)\right)$ is locally stable if $a T+\ln \left(1-p_{1}\right) \leq 0$.

(2) The periodic solution $\left(0,0, z^{*}(t)\right)$ is unstable if aT $+\ln \left(1-p_{1}\right)>0$.

Proof. (1) The local stability of the periodic solution $\left(0,0, z^{*}(t)\right)$ of the system (1.2) may be determined by considering the behavior of small amplitude perturbations 
of the solution. Let $(x(t), y(t), z(t))$ be any solution of the system (1.2). Define $x(t)=u(t), y(t)=v(t), z(t)=w(t)+z^{*}(t)$. Then they may be written as

$$
\left(\begin{array}{c}
u(t) \\
v(t) \\
w(t)
\end{array}\right)=\Phi(t)\left(\begin{array}{c}
u(0) \\
v(0) \\
w(0)
\end{array}\right)
$$

where $\Phi(t)$ satisfies

$$
\frac{d \Phi}{d t}=\left(\begin{array}{ccc}
a & 0 & 0 \\
0 & -d_{1}-c_{3} z^{*}(t) & 0 \\
0 & c_{4} z^{*}(t) & -d_{2}
\end{array}\right) \Phi(t)
$$

and $\Phi(0)=I$ is the identity matrix. So the fundamental solution matrix is

$$
\Phi(t)=\left(\begin{array}{ccc}
\exp (a t) & 0 & 0 \\
0 & \exp \left(\int_{0}^{t}-d_{1}-c_{3} z^{*}(s) d s\right) & 0 \\
0 & \exp \left(\int_{0}^{t} c_{4} z^{*}(s) d s\right) & \exp \left(-d_{2} t\right)
\end{array}\right) .
$$

The resetting impulsive conditions of the system (1.2) become

$$
\left(\begin{array}{l}
u\left((n+\tau-1) T^{+}\right) \\
v\left((n+\tau-1) T^{+}\right) \\
u\left((n+\tau-1) T^{+}\right)
\end{array}\right)=\left(\begin{array}{ccc}
1-p_{1} & 0 & 0 \\
0 & 1-p_{2} & 0 \\
0 & 0 & 1-p_{3}
\end{array}\right)\left(\begin{array}{c}
u((n+\tau-1) T) \\
v((n+\tau-1) T) \\
w((n+\tau-1) T)
\end{array}\right)
$$

and

$$
\left(\begin{array}{c}
u\left(n T^{+}\right) \\
v\left(n T^{+}\right) \\
w\left(n T^{+}\right)
\end{array}\right)=\left(\begin{array}{lll}
1 & 0 & 0 \\
0 & 1 & 0 \\
0 & 0 & 1
\end{array}\right)\left(\begin{array}{c}
u(n T) \\
v(n T) \\
w(n T)
\end{array}\right) .
$$

Note that the eigenvalues of

$$
S=\left(\begin{array}{ccc}
1-p_{1} & 0 & 0 \\
0 & 1-p_{2} & 0 \\
0 & 0 & 1-p_{3}
\end{array}\right)\left(\begin{array}{ccc}
1 & 0 & 0 \\
0 & 1 & 0 \\
0 & 0 & 1
\end{array}\right) \Phi(T)
$$

are $\mu_{1}=\left(1-p_{1}\right) \exp (a T), \mu_{2}=\left(1-p_{2}\right) \exp \left(-\int_{0}^{T} d_{1}+c_{3} z^{*}(s) d s\right)<1$ and $\mu_{3}=$ $\left(1-p_{3}\right) \exp \left(-d_{2} T\right)<1$. The condition $\mu_{1} \leq 1(>1)$ is equivalent to the equation $a T+\ln \left(1-p_{1}\right) \leq 0(>0)$. Therefore, by Lemma 2.8 , the periodic solution $\left(0,0, z^{*}(t)\right)$ is locally stable if $a T+\ln \left(1-p_{1}\right) \leq 0$ and is unstable if $a T+\ln \left(1-p_{1}\right)>0$.

Theorem 3.3. The periodic solution $\left(x^{*}(t), 0, z^{*}(t)\right)$ is locally asymptotically stable if $a T+\ln \left(1-p_{1}\right)>0$ and

$$
\int_{0}^{T} \frac{c_{2} x^{*}(t)}{1+e_{1}\left(x^{*}(t)\right)^{2}} d t+\ln \left(1-p_{2}\right)<d_{1} T+\Delta
$$


where $\Delta=\frac{c_{3} q\left(1+\left(p_{3}-1\right) \exp \left(-d_{2} T\right)-p_{3} \exp \left(-d_{2} \tau T\right)\right)}{d_{2}\left(1-\left(1-p_{3}\right) \exp \left(-d_{2} T\right)\right)}$.

Proof. Now, we apply the same method as Theorem 3.2 to the periodic solution $\left(x^{*}(t), 0, z^{*}(t)\right)$ to determine its stability. So, we define $x(t)=u(t)+x^{*}(t), y(t)=$ $v(t), z(t)=w(t)+z^{*}(t)$. Then they may be written as

$$
\left(\begin{array}{c}
u(t) \\
v(t) \\
w(t)
\end{array}\right)=\Phi(t)\left(\begin{array}{c}
u(0) \\
v(0) \\
w(0)
\end{array}\right)
$$

where $\Phi(t)$ satisfies

$$
\frac{d \Phi}{d t}=\left(\begin{array}{ccc}
a-2 b x^{*}(t) & -\frac{c_{1} x^{*}(t)}{1+e_{1}\left(x^{*}(t)\right)^{2}} & 0 \\
0 & -d_{1}-c_{3} z^{*}(t)+\frac{c_{2} x^{*}(t)}{1+e_{1}\left(x^{*}(t)\right)^{2}} & 0 \\
0 & c_{4} z^{*}(t) & -d_{2}
\end{array}\right) \Phi(t)
$$

and $\Phi(0)=I$ is the identity matrix. The resetting impulsive conditions of the system (1.2) become

$$
\left(\begin{array}{l}
u\left((n+\tau-1) T^{+}\right) \\
v\left((n+\tau-1) T^{+}\right) \\
u\left((n+\tau-1) T^{+}\right)
\end{array}\right)=\left(\begin{array}{ccc}
1-p_{1} & 0 & 0 \\
0 & 1-p_{2} & 0 \\
0 & 0 & 1-p_{3}
\end{array}\right)\left(\begin{array}{c}
u((n+\tau-1) T) \\
v((n+\tau-1) T) \\
w((n+\tau-1) T)
\end{array}\right)
$$

and

$$
\left(\begin{array}{c}
u\left(n T^{+}\right) \\
v\left(n T^{+}\right) \\
w\left(n T^{+}\right)
\end{array}\right)=\left(\begin{array}{lll}
1 & 0 & 0 \\
0 & 1 & 0 \\
0 & 0 & 1
\end{array}\right)\left(\begin{array}{c}
u(n T) \\
v(n T) \\
w(n T)
\end{array}\right)
$$

Further, the eigenvalues of

$$
S=\left(\begin{array}{ccc}
1-p_{1} & 0 & 0 \\
0 & 1-p_{2} & 0 \\
0 & 0 & 1-p_{3}
\end{array}\right)\left(\begin{array}{ccc}
1 & 0 & 0 \\
0 & 1 & 0 \\
0 & 0 & 1
\end{array}\right) \Phi(T)
$$

are $\mu_{1}=\left(1-p_{1}\right) \exp \left(\int_{0}^{T} a-2 b x^{*}(t) d t\right), \mu_{2}=\left(1-p_{2}\right) \exp \left(\int_{0}^{T}-d_{1}-c_{3} z^{*}(t)+\right.$ $\left.\frac{c_{2} x^{*}(t)}{1+e_{1}\left(x^{*}(t)\right)^{2}} d t\right)$ and $\mu_{3}=\left(1-p_{3}\right) \exp \left(-d_{2} T\right)<1$. Since $\int_{0}^{T} x^{*}(t) d t=\frac{1}{b}\left(\ln \left(1-p_{1}\right)+\right.$ $a T)$ and

$$
\int_{0}^{T} z^{*}(t) d t=\frac{q\left(1+\left(p_{3}-1\right) \exp \left(-d_{2} T\right)-p_{3} \exp \left(-d_{2} \tau T\right)\right)}{d_{2}\left(1-\left(1-p_{3}\right) \exp \left(-d_{2} T\right)\right)}
$$

we get that $\left|\mu_{1}\right|<1$ and the condition $\left|\mu_{2}\right|<1$ is equivalent to the equation (3.4). Therefore, from Lemma 2.8 , we obtain $\left(x^{*}(t), 0, z^{*}(t)\right)$ is locally asymptotically stable. 
Remark 3.4. (1) It is not easy to evaluate the value of $\int_{0}^{T} \frac{x^{*}(t)}{1+e_{1}\left(x^{*}(t)\right)^{2}} d t$. Actually, for $0<t<T$, we obtain $x^{*}(t)=\frac{a \eta \exp (a t)}{b(1-\eta+\eta \exp (a t))}$ and hence $\int \frac{x^{*}(t)}{1+e_{1}\left(x^{*}(t)\right)^{2}} d t=$ $\frac{a b c_{2} \eta \Delta}{2 b e_{1} \eta a^{3}+2 b^{3} \eta a}$, where $\Delta=\Delta_{1}-\Delta_{2}, \Delta_{1}=b \ln \left(a^{2} \exp (2 a t) e_{1} \eta^{2}+b^{2}(((\exp (a t)-1)+\right.$ $\left.\left.1)^{2}\right)\right), \Delta_{2}=2 a \sqrt{e_{1}} \tan ^{-1}\left(\frac{\exp (a t) e_{1} \eta a^{2}+b^{2}((\exp (a t)-1)+1)}{a b \sqrt{e_{1}}(\eta-1)}\right)$ and $\eta=\frac{\left(1-p_{1}\right) \exp (a T)-1}{\exp (a T)-1}$.

(2) We get Theorem 3.1 in [20] as a Corollary of Theorem 3.2.

Theorem 3.5. The following statements hold.

(1) The periodic solution $\left(0,0, z^{*}(t)\right)$ is globally asymptotically stable if aT $+\ln (1-$ $\left.p_{1}\right) \leq 0$.

(2) The periodic solution $\left(x^{*}(t), 0, z^{*}(t)\right)$ is globally asymptotically stable if aT + $\ln \left(1-p_{1}\right)>0$ and

$$
\frac{c_{2}}{b}\left(\ln \left(1-p_{1}\right)+a T\right)+\ln \left(1-p_{2}\right)<d_{1} T+\Lambda,
$$

where $\Lambda=\frac{c_{3} q\left(1+\left(p_{3}-1\right) \exp \left(-d_{2} T\right)-p_{3} \exp \left(-d_{2} \tau T\right)\right)}{\left(1+e_{1} M^{2}\right) d_{2}\left(1-\left(1-p_{3}\right) \exp \left(-d_{2} T\right)\right)}$ and $M$ is an ultimate boundedness constant for $y(t)$ in Theorem 3.1 .

Proof. (1) Assume that $a T+\ln \left(1-p_{1}\right) \leq 0$. It is easy to see from the proof of Theorem 3.2 that the periodic solution $\left(0,0, z^{*}(t)\right)$ is locally asymptotically stable. Take $\epsilon_{1}>0$ such that $\epsilon_{1}<\frac{d_{1}}{c_{2}}$. Let $\xi=\left(1-p_{1}\right) \exp \left(\left(c_{2} \epsilon_{1}-d_{1}\right) T\right)$. Then $0<\xi<1$. It is seen from the first equation in (1.2) that $x^{\prime}(t)=x(t)(a-b x(t))-\frac{c_{1} x(t) y(t)}{1+e_{1} x^{2}(t)} \leq$ $x(t)(a-b x(t))$ for $t \neq(n+\tau-1) T$. By Lemma 2.3, $x(t) \leq \tilde{x}(t)$ for $t \geq 0$, where $\tilde{x}(t)$ is the solution of (2.6) with $x_{0}>0$. From Lemma 2.6, we can choose $T_{1}>0$ satisfying $x(t) \leq \epsilon_{1}$ for $t \geq T_{1}$. Without loss of generality, we may assume that $x(t) \leq \epsilon_{1}$ for all $t>0$. We obtain from the second equation in (1.2) that, for $t \neq(n+\tau-1) T$,

$$
\begin{aligned}
y^{\prime}(t) & =-d_{1} y(t)+\frac{c_{2} x(t) y(t)}{1+e_{1} x^{2}(t)}-\frac{c_{3} y(t) z(t)}{1+e_{2} y^{2}(t)} \\
& \leq-d_{1} y(t)+c_{2} x(t) y(t) \\
& \leq y(t)\left(-d_{1}+c_{2} \epsilon_{1}\right) .
\end{aligned}
$$

Integrating (3.6) on $((n+\tau-1) T,(n+\tau) T]$, we get

$$
y((n+\tau) T) \leq y\left((n+\tau-1) T^{+}\right) \exp \left(\left(-d_{1}+c_{2} \epsilon_{1}\right) T\right)=y((n+\tau-1) T) \xi
$$

and hence $y((n+\tau) T) \leq y(\tau T) \xi^{n}$ which implies that $y((n+\tau) T) \rightarrow 0$ as $n \rightarrow \infty$. Further, we obtain that $y(t) \leq y\left((n+\tau-1) T^{+}\right) \exp \left(\left(-d_{1}+c_{2} \epsilon_{1}\right)(t-(n+\tau-1) T)\right) \leq$ $y((n+\tau-1) T)$ for $t \in((n+\tau-1) T,(n+\tau) T]$. Thus $y(t) \rightarrow 0$ as $n \rightarrow \infty$. Now, take $0<\epsilon_{2}<\frac{d_{2}}{c_{4}}$ to prove that $z(t) \rightarrow z^{*}(t)$ as $t \rightarrow \infty$. Since $\lim _{t \rightarrow \infty} y(t)=0$, there is a $T_{2}>0$ that $y(t) \leq \epsilon_{2}$ for $t \geq T_{2}$. For the sake of simplicity, we assume that $y(t) \leq \epsilon_{2}$ 
for all $t$. It follows from the third equation in (1.2) that, for $t \neq(n+\tau-1) T, t \neq n T$,

$$
\begin{aligned}
-d_{2} z(t) \leq z^{\prime}(t) & =-d_{2} z(t)+\frac{c_{4} y(t) z(t)}{1+e_{2} y^{2}(t)} \\
& \leq-\left(d_{2}-c_{4} \epsilon_{2}\right) z(t) .
\end{aligned}
$$

Thus, it is induced from Lemma 2.3 that $\tilde{z}_{1}(t) \leq z(t) \leq \tilde{z}_{2}(t)$, where $\tilde{z}_{1}(t)$ is the solution of $(2.8)$ and $\tilde{z}_{2}(t)$ is also the solution of (2.8) with $d_{2}$ changed into $d_{2}-c_{4} \epsilon_{2}$. Therefore, it follows from Lemma 2.7 and taking sufficiently small $\epsilon_{2}>0$ that $\tilde{z}_{1}(t)$ and $\tilde{z}_{2}(t)$ tend to $z^{*}(t)$ as $t \rightarrow \infty$. Thus we get $\left|z(t)-z^{*}(t)\right| \rightarrow 0$ as $t \rightarrow \infty$.

(2) Suppose that $a T+\ln \left(1-p_{1}\right)>0$ and (3.5) hold. Then, by (3.5), we can choose $\epsilon_{3}>0$ such that

$$
0<\zeta \equiv\left(1-p_{2}\right) \exp \left(-d_{1} T+c_{2}\left(\frac{1}{b}\left(\ln \left(1-p_{1}\right)+a T\right)+\epsilon_{3} T\right)-\Theta\right)<1,
$$

where $\Theta=\frac{c_{3} q\left(1+\left(p_{3}-1\right) \exp \left(-d_{2} T\right)-p_{3} \exp \left(-d_{2} \tau T\right)\right)}{\left(1+e_{2} M^{2}\right) d_{2}\left(1-\left(1-p_{3}\right) \exp \left(-d_{2} T\right)\right)}+\epsilon_{3} T$. From Theorem 3.1, without loss of generality, we may assume that $x(t), y(t)<M$ for all $t$. As in the proof of (1), by Lemma $2.3, x(t) \leq \tilde{x}_{2}(t)$ is obtained for $t \geq 0$, where $\tilde{x}_{2}(t)$ is the solution of (2.6). It is from Lemma 2.6 that there exists a $T_{3}>0$ such that $x(t) \leq x^{*}(t)+\epsilon_{3}$ for $t \geq T_{3}$. Without loss of generality, we may assume that $x(t) \leq x^{*}(t)+\epsilon_{3}$ for $t>0$. Since $z^{\prime}(t)=-d_{2} z(t)+\frac{c_{4} y(t) z(t)}{1+e_{2} y^{2}(t)} \geq-d_{2} z(t)(t \neq(n+\tau-1) T, t \neq n T)$, by Lemma 2.3, $z(t) \geq \tilde{z}(t)$ is obtained for $t>0$, where $\tilde{z}(t)$ is the solution of (2.8). From Lemma 2.7, we can select a $T_{4}>0$ satisfying $z(t) \geq z^{*}(t)+\epsilon_{3}$ for $t \geq T_{4}$. For the sake of simplicity, we suppose that $z(t) \geq z^{*}(t)+\epsilon_{3}$ for $t>0$. From the second equation in (1.2), we obtain that, for $t \neq(n+\tau-1) T$,

$$
\begin{aligned}
y^{\prime}(t) & =-d_{1} y(t)+\frac{c_{2} x(t) y(t)}{1+e_{1} x^{2}(t)}-\frac{c_{3} y(t) z(t)}{1+e_{2} y^{2}(t)} \\
& \leq y(t)\left(-d_{1}+c_{2}\left(x^{*}(t)+\epsilon_{3}\right)-\frac{c_{3}}{1+e_{2} M^{2}}\left(z^{*}(t)+\epsilon_{3}\right)\right) .
\end{aligned}
$$

By integrating (3.8) on $((n+\tau-1) T,(n+\tau) T]$, we obtain that $y((n+\tau) T) \leq$ $y((n+\tau-1) T+) \exp \left(\int_{(n+\tau-1) T^{+}}^{(n+\tau) T}-d_{1}+c_{2}\left(x^{*}(t)+\epsilon_{3}\right)-\frac{c_{3}}{1+e_{2} M^{2}}\left(z^{*}(t)+\epsilon_{3}\right) d t\right)=$ $y((n+\tau-1) T) \zeta$. Thus, $y((n+\tau) T) \leq y(\tau T) \zeta^{n}$ which implies that $y((n+\tau) T) \rightarrow 0$ as $n \rightarrow \infty$. Further, the inequality $y^{\prime}(t) \leq c_{2} x(t) y(t) \leq c_{2} M y(t)$ implies that $y(t) \leq$ $\left(1-p_{2}\right) y((n+\tau-1) T) \exp \left(c_{2} M T\right)$, for $t \in((n+\tau-1) T,(n+\tau) T]$, and consequently $y(t) \rightarrow 0$ as $t \rightarrow \infty$. Now, let $0<\epsilon_{4}<\frac{a}{c_{1}}$ to show that $\left|x(t)-x^{*}(t)\right| \rightarrow 0$ as $t \rightarrow \infty$. Since $y(t) \rightarrow 0$ as $t \rightarrow \infty$, there exists a $T_{5}>0$ such that $y(t)<\epsilon_{4}$ for $t>T_{5}$. For the sake of simplicity, we suppose that $y(t)<\epsilon_{4}$ for all $t>0$. It follows that, for $t \neq(n+\tau-1) T, t \neq n T$,

$$
\begin{aligned}
x^{\prime}(t) & =x(t)(a-b x(t))-\frac{c_{1} x(t) y(t)}{1+e_{1} x^{2}(t)} \\
& \geq x(t)\left(\left(a-c_{1} \epsilon_{4}\right)-b x(t)\right) .
\end{aligned}
$$


Thus, it follows from Lemma 2.3 that $\tilde{x}_{1}(t) \leq x(t)$, where $\tilde{x}_{1}(t)$ is the solution of (2.6) with $a$ changed into $a-c_{1} \epsilon_{4}$. From Lemma 2.6 and taking sufficiently small $\epsilon_{4}>0$, we see that $\tilde{x}_{1}(t)$ and $\tilde{x}_{2}(t)$ tend to $x^{*}(t)$ as $t \rightarrow \infty$. Thus, we get $\left|x(t)-x^{*}(t)\right| \rightarrow 0$ as $t \rightarrow \infty$. By using the same process as the proof of (1), we can show that $\left|z(t)-z^{*}(t)\right| \rightarrow 0$ as $t \rightarrow \infty$.

\section{References}

[1] R. Arditi and L. R. Ginzburg, Coupling in predator-prey dynamics:Ratio-dependence, J. Theor. Biol., 139(1989), 311-326.

[2] D. D. Bainov and P. S. Simeonov, Impulsive Differential Equations:Periodic Solutions and Applications, vol. 66, of Pitman Monographs and Surveys in Pure and Applied Mathematics, Longman Science \& Technical, Harlo, UK, 1993.

[3] J. B. Collings, The effects of the functional response on the bifurcation behavior of a mite predator-prey interaction model, J. Math. Biol., 36(1997), 149-168.

[4] C. Cosner, D. L. DeAngelis, Effects of spatial grouping on the functional response of predators, Theoretical Popuation Biology, 56(1999), 65-75.

[5] H. I. Freedman and R. M. Mathsen, Persistence in predator-prey systems with ratiodependent predator influence, Bulletin of Math. Biology, 55(4)(1993), 817-827.

[6] M. P. Hassell and G. C. Varley, New inductive population model for insect parasites and its bearing on biological control, Nature, 223(1969), 1133-1136.

[7] S.-B. Hsu and T.-W. Huang, Global stability for a class of predator-prey systems, SIAM J. Appl. Math., 55(3)(1995), 763-783.

[8] K. Kitamura, K. Kashiwagi, K.-i. Tainaka, T. Hayashi, J. Yoshimura, T. Kawai and T. Kajiwara, Asymmetrical effect of migration on a prey-predator model, Physics Letters A, 357(2006), 213-217.

[9] V Lakshmikantham, D. Bainov, P.Simeonov, Theory of Impulsive Differential Equations, World Scientific Publisher, Singapore, 1989.

[10] B. Liu, Z. Teng and L. Chen, Analsis of a predator-prey model with Holling II functional response concerning impulsive control strategy, J. of Comp. and Appl. Math., 193(1)(2006), 347-362

[11] B. Liu, Y. J. Zhang, L. S. Chen and L. H. Sun, The dynamics of a prey-dependent consumption model concerning integrated pest management, Acta Mathematica Sinica, English Series, 21(3)(2005), 541-554.

[12] X. Liu and L. Chen, Complex dynamics of Holling type II Lotka-Volterra predator-prey system with impulsive perturbations on the predator, Chaos, Solitons and Fractals, 16(2003), 311-320.

[13] P. Georgescu and G. Morosanu, Impulsive perturbations of a three-trophic prey-dependent food chain system, Mathematical and Computer Modeling(2008), doi:10.1016/j.mcm.2007.12.006. 
[14] S. Ruan and D. Xiao, Global analysis in a predator-prey sytem with non-monotonic functional response, SIAM J. Appl. Math., 61(4)(2001), 1445-1472.

[15] E, Saez and E. Gonzalez-Olivares, Dynamics of a predator-prey model, SIAM J. Appl. Math., 59(5)(1999), 1867-1878.

[16] G. T. Skalski and J. F. Gilliam, Funtional responses with predator interference: viable alternatives to the Holling type II mode, Ecology, 82(2001), 3083-3092.

[17] W. Wang, H. Wang and Z. Li, Chaotic behavior of a three-species Beddington-type system with impulsive perturbations, Chaos Solitons and Fractals, 37(2008), 438-443.

[18] S. Zhang and L. Chen, Chaos in three species food chain system with impulsive perturbations, Chaos Solitons and Fractals, 24(2005), 73-83.

[19] S. Zhang and L. Chen, A Holling II functional response food chain model with impulsive perturbations, Chaos Solitons and Fractals, 24(2005), 1269-1278.

[20] S. Zhang, F. Wang and L. Chen, A food chain model with impulsive perturbations and Holling IV functional response, Chaos, Solitons and Fractals, 26(2005), 855-866.

[21] Y. Zhang, B. Liu and L. Chen, Extinction and permanence of a two-prey one-predator system with impulsive effect, Mathematical Medicine and Biology, 20(2003), 309-325. 